\title{
AN INTERACTIVE DESIGN SYSTEM FOR HYBRID ICs
}

\author{
HIROSHI SHIRAISHI $\dagger$, KAORU KAWAMURA ${ }^{\dagger}$ and MASAAKI HAYASHI $\ddagger$ \\ †Computer-Based Systems Lab., Fujitsu Laboratories Ltd., \\ ¥Transmission Division, Fujitsu Ltd., \\ 1015 Kamikodanaka, Nakaharaku, Kawasaki, Japan
}

\begin{abstract}
In hybrid integrated circuit mask design, the design quality largely depends on the designer's experience and creativity. It is difficult to completely automate the design process as is done with LSIs or printed circuit boards. It is important to incorporate flexibility into the system.

This paper describes an interactive design system for laying out hybrid ICs. The system is implemented on a large host computer, and graphic display terminals are connected to it in a time sharing environment. The system provides designers with many interactive facilities which are useful in designing hybrid ICs. In addition to highly interactive dialogue, the system has capabilities such as dynamic checking of the designer's operations against a set of design rules, or automatic generation of standardized element patterns.

The system outputs mask information, design documents and so on which are used in the CAM of hybrid ICs. The system is being used at Fujitsu and is contributing to high reliability of the design, and shortening the design period.
\end{abstract}

\section{INTRODUCTION}

The CAD (Computer Aided Design) techniques have been applied to design masks for high-quality thin-film HICs (Hybrid Integrated Circuits) used as high-frequency circuits. There are two main approaches in applying CAD technique to HIC design.

One approach aims for completely-automatic HIC design and attempts to automate the entire HIC design procedure through algorithmic methods such as circuit planarization, placement, and inter-connection. ${ }^{1-3}$ Although complete automation has been successfully applied to LSIs or printed circuit boards, its application to HICs is difficult for the following reasons:

HICs have various kind of element patterns.

Automatic placement and routing would affect the electric characteristics.

HIC design depends to a large extent on the experience and creativity of the designer.

Therefore, an interactive system should be developed so the designer's ideas may be immediately incorporated into HIC design.

The other approach is based on the interactive design concept, and HICs are designed interactively using a graphical input/output terminal such as graphic display unit. ${ }^{4,5}$ The system described in this paper was developed in line with this approach, but it has several unique characteristics not shared by other interactive systems, as detailed in the following sections.
This paper describes the IDLE-H (Interactive $D$ esign Aids for Laying-out of Electrical Circuits $H$ ybrid IC) system used to design HIC masks interactively. Section 2 explains the design philosophy of the system, to make clear how IDLE-H differs from other interactive design systems. Section 3 explains how IDLE-H has been implemented, and shows the configuration of the system. Section 4 describes several examples using IDLE-H.

The IDLE-H system is currently in use at Fujitsu to improve design reliability and reduce total design time.

\section{BACKGROUND - SYSTEM DESIGN PHILOSOPHY}

This section describes the basic philosophy in the design of the IDLE-H system.

At first, let's review HIC design procedure. As shown in Figure 1, the first step is to design a circuit to satisfy the specifications. When the circuit is designed, both the values of elements in the circuit and the connections between elements are determined. The designer designs element patterns from the determined values. He then locates the elements on the HIC substrate and interconnects them with conductors. During placement and interconnection, he checks the pattern effectiveness and the density on the substrate If necessary, he re-designs element patterns or replaces elements. After elements are placed and interconnected, the designer checks the entire HIC 


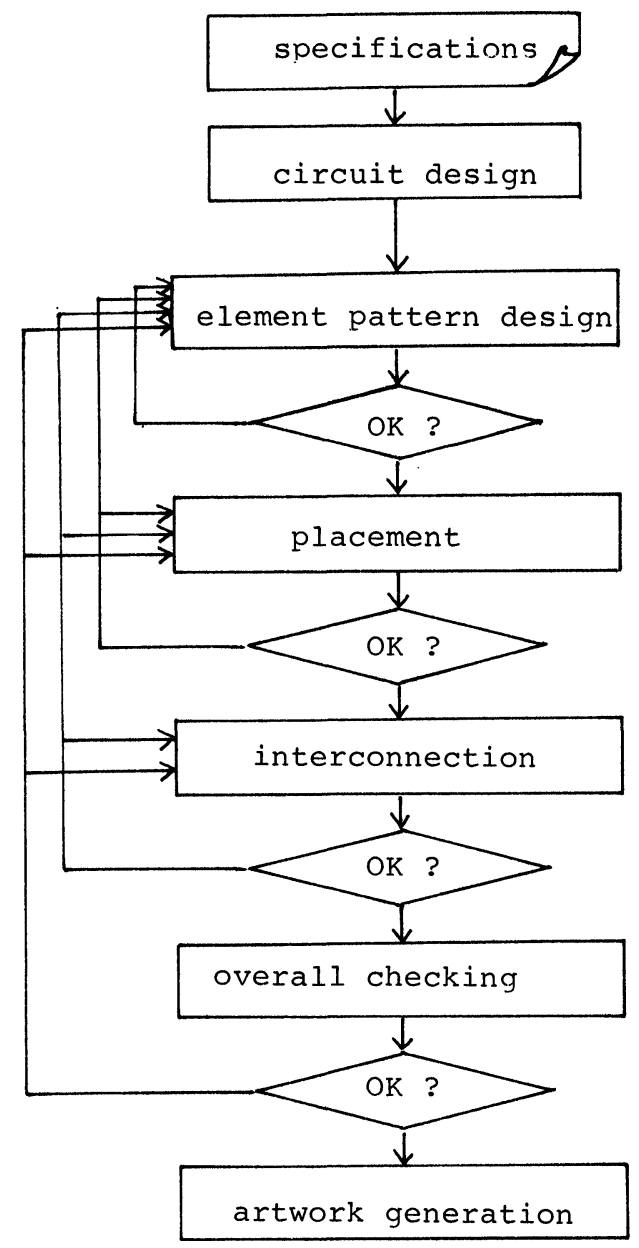

(a)

FIGURE 1 HIC design procedure.

design. If there are no problems, art-work generation can be started.

It is clear that an interactive input system is not enough to support the entire HIC design procedure described so far. Most conventional interactive design systems can be used only in step (c) and (e) in Figure 1. To substantially reduce total design time, the entire procedure (step (a) through (h)) had to be supported.

The ideal interactive design system is:

A system that can perform the entire HIC design procedure interactively, so that the designer needs only a rough layout image in his mind and circuit schematics in his hand, when he sits in front of the graphic display terminal.

HIC design procedure steps are not always sequentially executed, as mentioned above. For example, the designer may want to re-design element patterns after placing them on the substrate. That is, a flexible system that allows steps to be performed in any order and any number of times is desirable. In addition, interactive commands provided by the system should be looked upon as being at the same level for the designer, and he should be allowed to issue these commands in any order.

Since HICs have many element patterns and high precision is required, it is important to develop a HIC design system which can ensure that the resultant design is correct. In most conventional interactive systems, this checking operation is performed by a Design Rule Check module after the HIC has been designed. In this checking mode, however, errors can be detected only after the entire HIC is designed. In addition, there is a possibility of accumulating errors in interactive design process. Therefore, a system that can perform design rule checking synchronized with each operation to ensure validity is desirable. ${ }^{6}$ The system should also provide high generality, i.e., a system permitting parameters (such as the maximum permissible spacing value) to be modified externally. Furthermore, the system should be able to correct the input pattern to satisfy design specifications when errors are detected during dynamic design rule checking.

Thin film elements on HICs are created by repeating evaporation, spattering, and etching procedures. Since high precision is required for masks used in these procedures, it is hard to design element mask patterns manually. In the IDLE-H system, the entire element mask pattern design operation was automated by standardizing the element design specifications in cooperation with the Design and Manufacturing Department.

\section{IMPLEMENTATION}

This section explains how IDLE-H was implemented, its hardware and software configurations, in line with the basic ideas described in section 2 .

\subsection{Hardware}

Figure 2 shows the IDLE-H hardware configuration.

The IDLE-H system is implemented under the TSS (Time Sharing System) environment using a large-scale FACOM M-180 II as the host computer. A graphic display terminal is connected to $\mathrm{M}-180 \mathrm{II}$ through a high-speed (9,600 baud) communication line. A Tektronix 4014 is currently used as the graphic display terminal. A high-resolution color display unit will be 


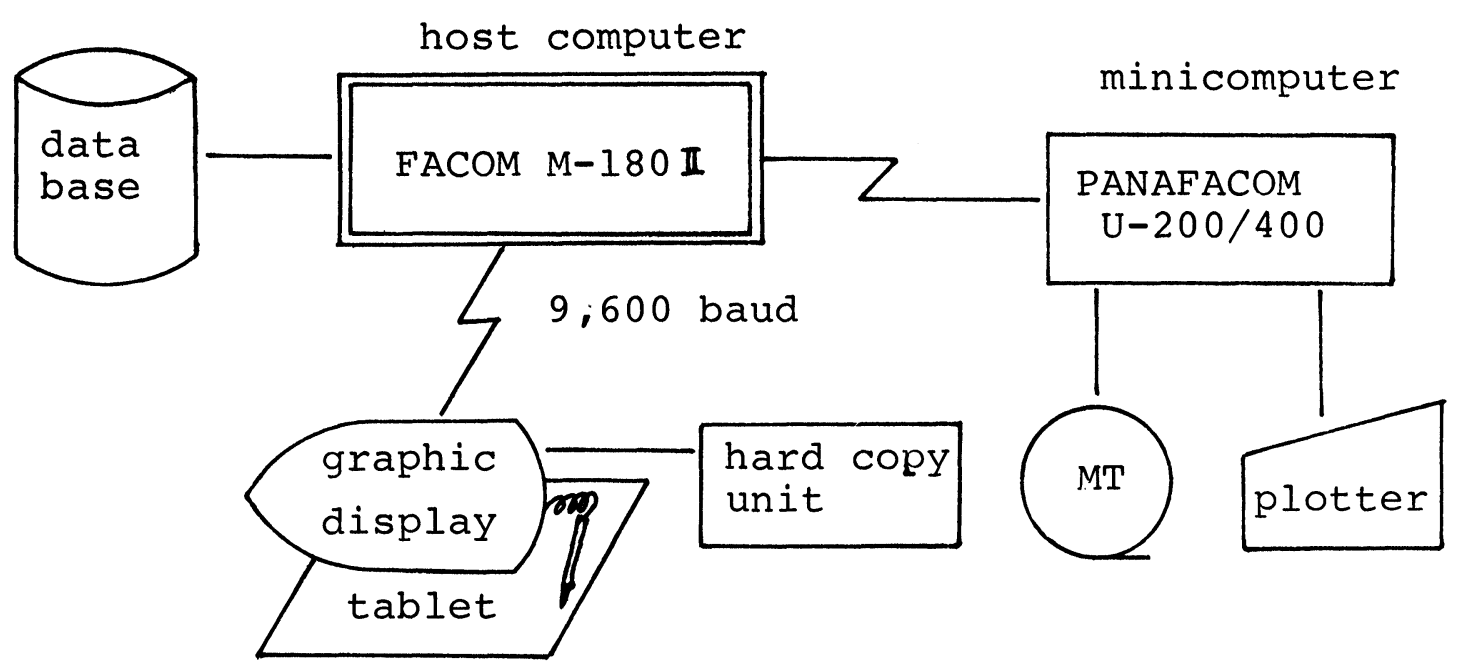

FIGURE 2 Hardware configuration.

installed in the near future. Communications between the designer and IDLE-H are enabled through commands entered on the tablet. The data during design and the design and the design results are stored in a data base in the host computer. After the mask design is completed, the results are sent to the PANAFACOM U-200 or U-400 minicomputer through the communication line. The minicomputer creates magnetic tapes for art-work generation and design documents for the production Department.

The advantages of connecting the graphic display terminal to a large computer through a high-speed communication line in a TSS environment are as follows:
Response time is reduced (the most important factor in interactive graphics).

Functions not easily supported by microcomputers can be implemented, such as automatic element pattern generation, dynamic design rule checking or geometric operations.

System development is facilitated by using the resources of the large computer. The IDLE-H system was developed in 1.5 man-years.

\subsection{Software}

Figure 3 shows the software configuration of the IDLE-H system.

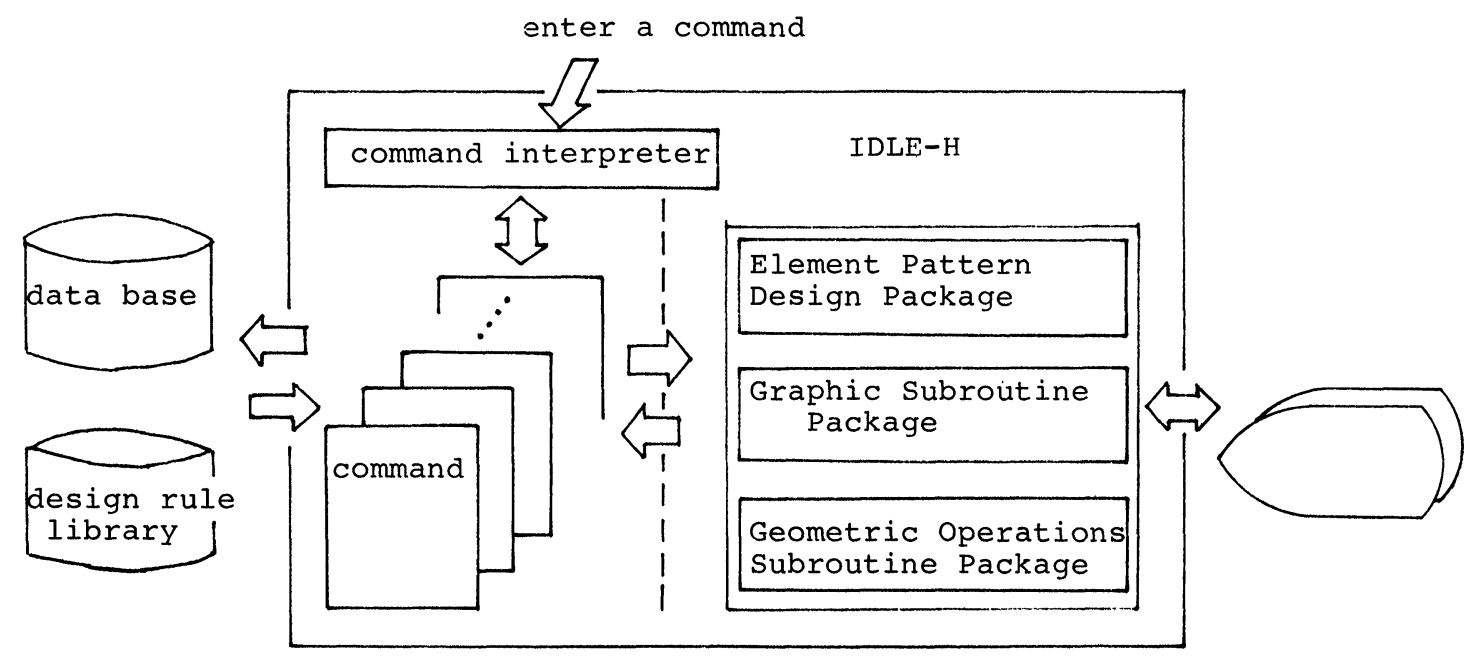

FIGURE 3 Software configuration. 
TABLE I

IDLE-H commands.

Command Name

Function

\begin{tabular}{|c|c|}
\hline CIRCUIT & Load data from the data base \\
\hline SAVE & Register the design results \\
\hline CONDITION & Change design rules \\
\hline MODE & Specify the checking mode \\
\hline PANEL & $\begin{array}{l}\text { Define and modify substrate } \\
\text { and external terminals }\end{array}$ \\
\hline ELEMENT & $\begin{array}{l}\text { Input and modify element da- } \\
\text { ta }\end{array}$ \\
\hline DISPLAY & Display \\
\hline ZOOM & Zoom (change screen) \\
\hline SHIFT & Shift the view \\
\hline GRID & Display grids \\
\hline RULER & Display ruler \\
\hline HARDCOPY & $\begin{array}{l}\text { Print monitor map on the } \\
\text { hard copy unit }\end{array}$ \\
\hline LIST & Listing data \\
\hline NEXT & $\begin{array}{l}\text { Select for next unconnected } \\
\text { net }\end{array}$ \\
\hline PLACE & $\begin{array}{l}\text { Specify and modify element } \\
\text { location }\end{array}$ \\
\hline$A D D$ & Add conductors \\
\hline DELETE & $\begin{array}{l}\text { Delete element.s and conduc- } \\
\text { tors }\end{array}$ \\
\hline MOVE & Move elements and conductors \\
\hline JOIN & $\begin{array}{l}\text { Combine overlapping patterns } \\
\text { into one (logical OR) }\end{array}$ \\
\hline HELP & List command menu \\
\hline MASK & Display masks \\
\hline COMMENT & Input comments \\
\hline CHECK & Overall checking \\
\hline COPV & $\begin{array}{l}\text { Copy the circuit to another } \\
\text { file }\end{array}$ \\
\hline FDELETE & Delete the circuit \\
\hline END & Terminate IDLE-H \\
\hline
\end{tabular}

When a command is entered by the designer, the command interpreter interprets it, requests the designer to input parameters if necessary, and passes control to the command program corresponding to the command. IDLE-H processes about 30 commands (listed in Table I.).

The design rule library contains parameters used for design rule checking, which may be changed by the designer. The data base stores both the data during design and the design results, so previously designed HICs may be re-designed easily.

The IDLE-H system also provides three sub-routine packages: geometric operations, graphic, and element pattern design.

3.2.1 Geometric operation subroutine package One of the major targets in developing the IDLE-H system was to provide design rule checking syncronized with each of the designer's operations. To enable the system to check many HIC patterns immediately, the geometric operations ${ }^{7}$ sub-routine package with high

TABLE II

Geometric operations.

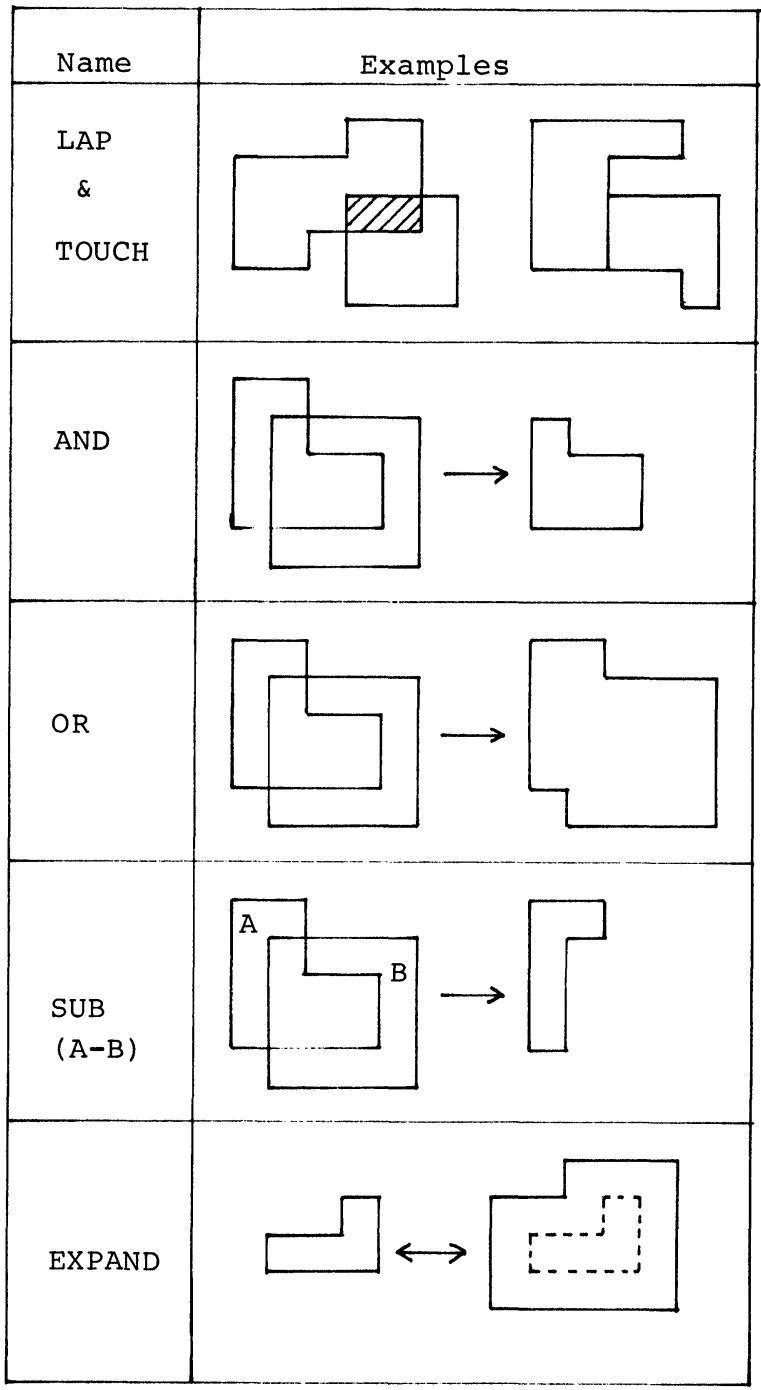


generality was developed. In this package the geometric operation subroutines explained in Table II are used to check and modify patterns. With these functions, the designer need not worry about violating the design rules even in designing high density HIC substrates.

3.2.2 Graphic subroutine package In recent years, performance of graphic display terminals has been greatly improved, and their price has been lowered. Accordingly, many types of graphic terminals are on the market. However, these terminals use different types of basic software, so that a great deal of software conversion is required to change to another display terminal. ${ }^{8}$

To solve this problem and achieve a device-independent system, the graphic subroutine package was developed. It makes the interface between the package and user program (IDLE-H) completely independent of the display device.

\subsubsection{Element pattern design subroutine}

package The element pattern design subroutine package is used to automatically generate element mask patterns. When the designer has entered data required for element mask design (such as the element value, current value, or precision) the sub-routines design the masks according to the element pattern design standards and return element mask pattern data (coordinate data). These subroutines can process resistors, ${ }^{9}$ capacitors, and coils as thin-film elements and can generate lands to which such discrete parts as transistors, diodes, chip condensers, and so on are to be attached.

\section{EXAMPLES}

This section gives several examples considered characteristic of the IDLE-H system element mask pattern design and conductor input. A sample HIC designed using the system follows the examples.

\subsection{Element Mask Pattern Design}

Mask patterns are designed by the element pattern design subroutine package. The designer needs only to input data in the following format:

$$
\mathrm{R} 1 \quad 1-2=1200,1,20 / \mathrm{T}=3
$$

where:

R1: Element name

1-2: Interconnection data (net list)
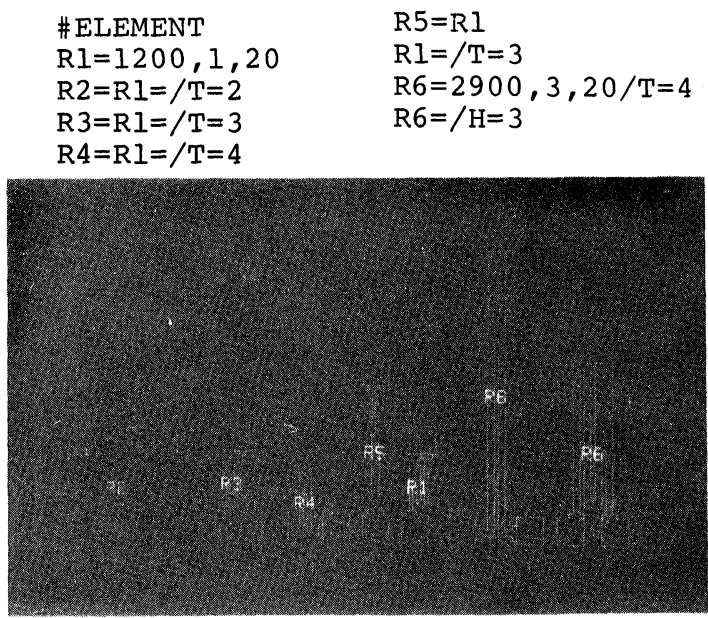

FIGURE 4 Mask pattern of resistor.

1200,1,20: Element parameters. In this example used for resistor mask design, 1200 is the resistor value (in $\Omega$ ), 1 is the current value (in $\mathrm{mA}$ ), and 20 is the precision (in \%).

$\mathrm{T}=3$ : Design parameters. These specify the design conditions such as the pattern type, or proportion of the vertical and horizontal lengths. When omitted, default values are assumed. In this example, $\mathrm{T}=3$ specifies the zigzag pattern.

When the data is input, the package designs the mask pattern and displays the results on the screen. The designer may obtain different patterns by modifying the design parameters. Refer to Figure 4.

The element pattern design package can also design capacitors, coils, and generate lands for discrete parts. Refer to Figure 5.

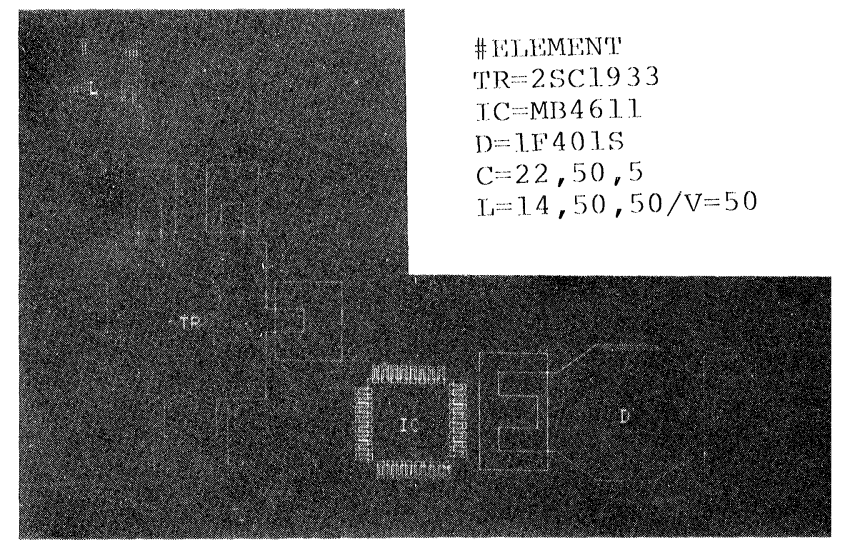

FIGURE 5 Element patterns. 


\subsection{Conductor Inputting}

One of the most important characteristics of the IDLE-H system is use of the dynamic design rule checking facility. This enables the designer to ensure correct operation while designing HICs. IDLE-H also provides a function which modifies the input pattern appropriately.

The dotted lines in Figure 6 (a) indicate the

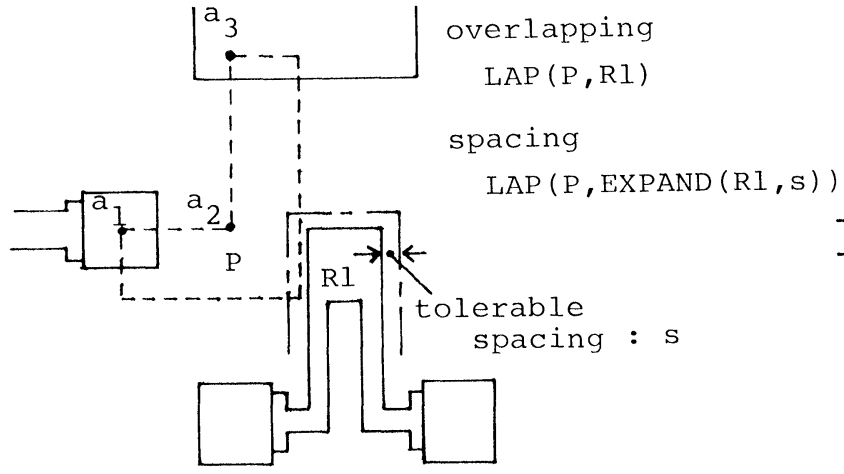

(a)

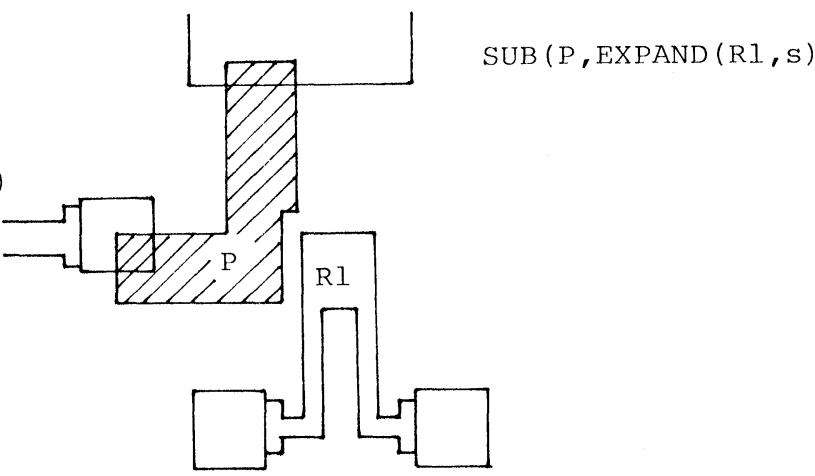

(b)

FIGURE 6 Design rule checking and automatic correction.

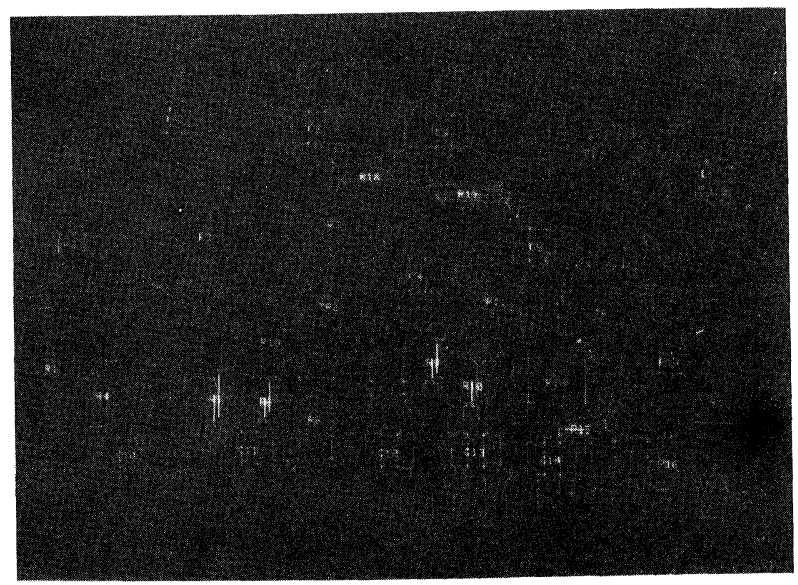

(a) Monitor Map on the Screen

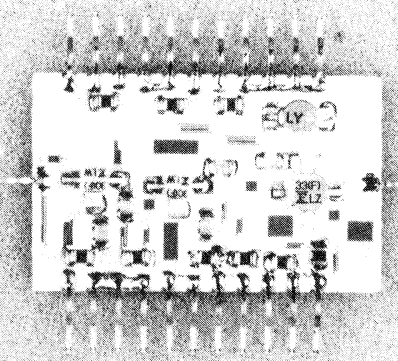

(c) $\mathrm{HIC}$
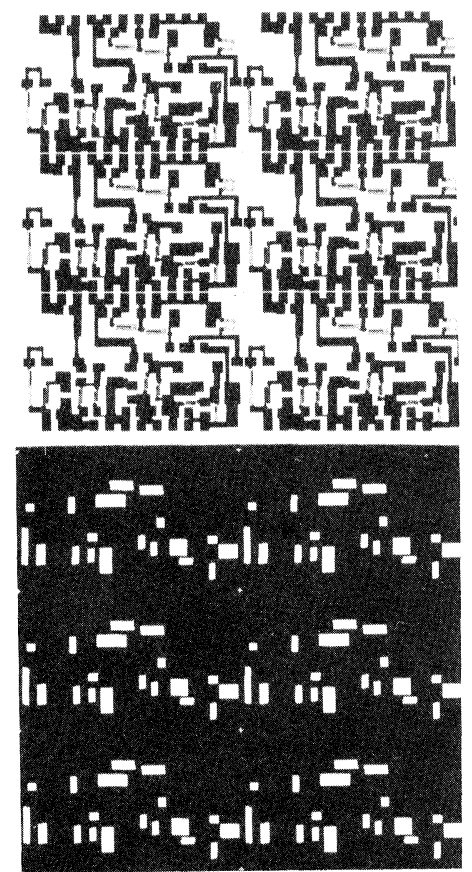

(b) Photo Masks

FIGURE 7 Example. 
standards. It uses the LAP to check overlapping and conductor pattern $\mathbf{P}$ input by the designer digitizing points $a_{1}, a_{2}$, and $a_{3}$ on the tablet. The IDLE-H system determines whether the pattern conforms to the design the EXPAND and LAP to check spacing. Since the distance between the input conductor and element R1 is out of the range specified by the standards in the example, IDLE-H issues an error message asking the designer whether it should correct the pattern. If the designer answers YES, IDLE-H automatically modifies the pattern so to satisfy the design standards and displays the modified pattern. During corrective modification, the system enlarges R1 using EXPAND, then cuts the input conductor $\mathbf{P}$ off by the enlarged $\mathrm{R} 1$ using SUB (Figure 6 (b)).

\subsection{HIC Example}

Figure 7 shows an HIC designed by the IDLE-H system on a $35 \mathrm{~m} / \mathrm{m} \times 22.5 \mathrm{~m} / \mathrm{m}$ substrate. a) is the layout displayed on the screen. Since this is a simple HIC example, it only took 2 hours to design from a rough layout sketch. b) is the HIC masks, and c) is the HIC picture taken after all parts are mounted.

\section{SUMMARY}

The system described in this paper, IDLE-H, provides the following advantages over other systems.

It can perform the entire HIC mask design interactively.

It can automatically generate element mask patterns. It uses the dynamic design rule checking function. It uses the automatic pattern correction function.
The IDLE-H system is now used at Fujitsu, and designers say it requires only half the design time required by conventional turn-key systems.

\section{ACKNOWLEDGEMENTS}

Studies on automatic HIC design system made by Messrs. T. Kojima, M. Yanagi, T. Uehara and $\mathrm{O}$. Takahashi furnished us with useful information. We also thank Messrs. T. Yamashita, S. Sasaki and M. Ishii for giving us the opportunity to study the system, and those who made great efforts in HIC design criteria standardization.

\section{REFERENCES}

1. T. Kojima, M. Yanagi and T. Uehara, "Computer-Aided Design of Hybrid IC Mask”, Fujitsu Sci. Tech. J., Vol. 10, No. 1 pp 75-93 (1974).

2. T. Uehara, H. Shiraishi, O. Takahashi and T. Kojima, "Embedding a Graph in a Plane with Local Constraints", IEEE ISCAS pp. 181-185, (1974).

3. K. Zibert and R. Saal, "On Computer Aided Hybrid Circuit Layout”, IEEE ISCAS, pp. 314-318, (1974).

4. N. Weste, "A Color Graphics System for IC Mask Design and Analysis", Proc. 15th D.A. Conf., pp. 199-205, (1978).

5. J. J. Degan, "Focus on Efficiency: Computer Graphics for HIC Design”, Bell Lab. Rec., pp. 286-292, (1974).

6. T. C. Bennett, K. R. Stevens and W. M. van Cleemput, "Dynamic Design Rule Checking in an Interactive Printed Circuit Editor", Proc. 16th D.A. Conf., pp. 330-336, (1979).

7. B. W. Lindsay and B. T. Preas, "Design Rule Checking and Analysis of IC Mask Designs", Proc. 13th D.A. Conf., pp. 301-308, (1976).

8. E. Hörbst, R. Prechtl and B. Will, "Engagement of Interactive Graphic Tools in a CAD-System for Digital Units", Proc. 13th D.A. Conf., pp. 86-90, (1976).

9. R. W. Berry, P. M. Hall and M. T. Harris, Thin-Film Technology, van Nostrand (1968). 

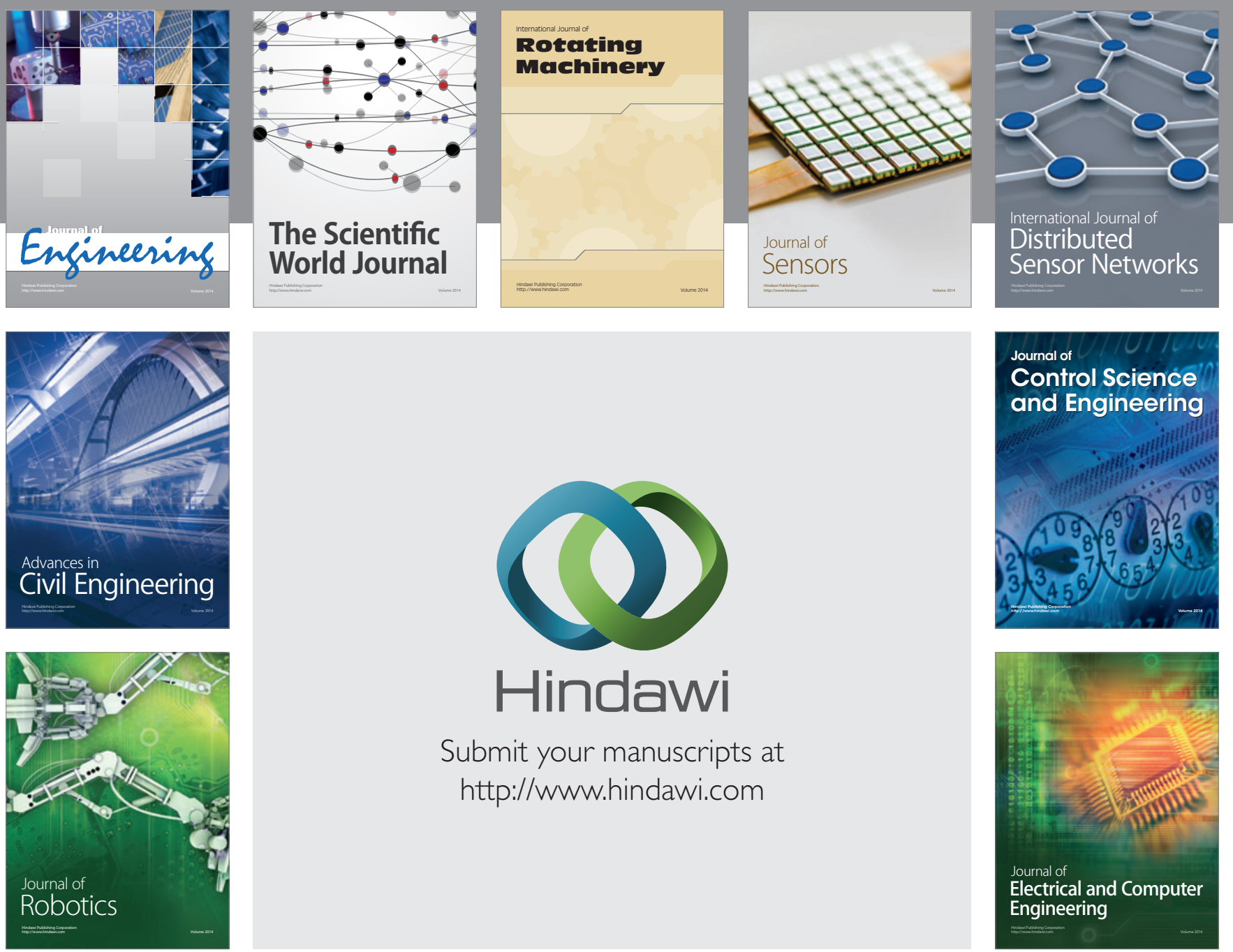

Submit your manuscripts at

http://www.hindawi.com
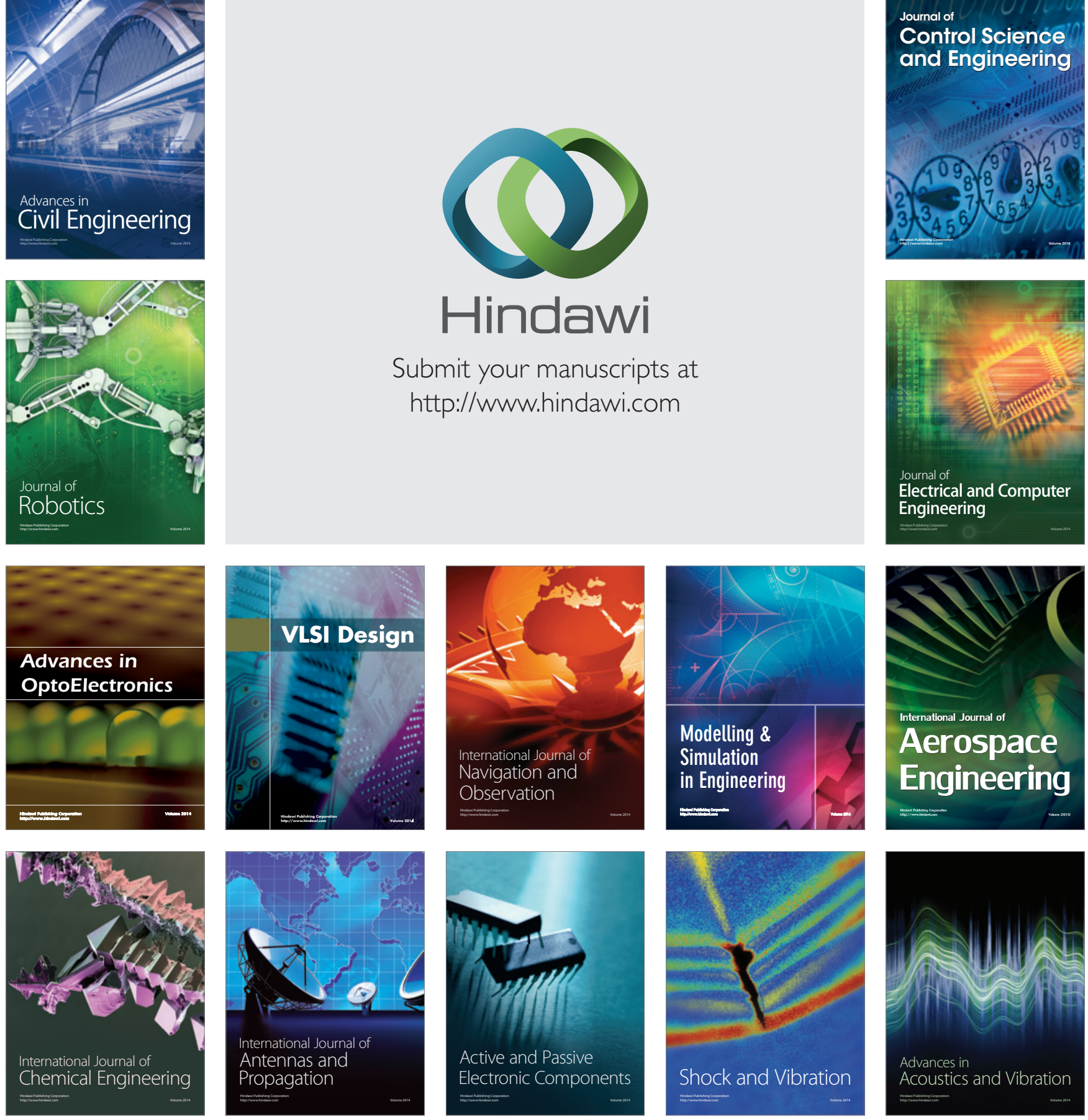